\title{
The specific frequencies of ultra-compact dwarf galaxies
}

\author{
S. Mieske ${ }^{1}$, M. Hilker ${ }^{2}$, and I. Misgeld ${ }^{2}$ \\ ${ }^{1}$ European Southern Observatory, Alonso de Cordova 3107, Vitacura, Santiago, Chile \\ e-mail: smieske@eso.org \\ ${ }^{2}$ European-Southern Observatory, Karl-Schwarzschild Str. 2, 85748 Garching, Germany
}

Received 5 July 2011 / Accepted 17 November 2011

\begin{abstract}
Context. One formation channel discussed for ultra-compact dwarf galaxies (UCDs) is that of massive star clusters, and the other main scenario is that of tidally transformed dwarf galaxies.

Aims. We aim at quantifying the specific frequency of UCDs in a range of environments and at relating this to the frequency of star clusters and potential progenitor dwarf galaxies. Are the frequencies of UCDs consistent with being the bright tail of the globular cluster luminosity function (GCLF)?

Methods. We propose a definition for the specific frequency of UCDs, $S_{\mathrm{N}, \mathrm{UCD}}=N_{\mathrm{UCD}} 10^{0.4\left(M_{V, \text { host }}-M_{V, 0}\right)} c_{\mathrm{w}}$. The parameter $M_{V, 0}$ is the zero point of the definition, chosen such that the specific frequency of UCDs is the same as those of globular clusters, $S_{\mathrm{N}, \mathrm{GC}}$, if UCDs follow a simple extrapolation of the GCLF. Considering UCDs as compact stellar systems with $M_{V}<-10.25$ mag (mass above $\sim 2 \times 10^{6} M_{\odot}$ ), it is $M_{V, 0}=-20 \mathrm{mag}$. The parameter $c_{\mathrm{w}}$ is a correction term to take the dependence of the GCLF width $\sigma$ on the host galaxy luminosity into account. We apply our definition of $S_{\mathrm{N}, \mathrm{UCD}}$ to results of spectroscopic UCD searches in the Fornax, Hydra, and Centaurus galaxy clusters, two Hickson compact groups, and the Local Group. This includes a large database of 180 confirmed UCDs in Fornax.

Results. We find that the specific frequencies derived for UCDs match those of GCs very well, to within $10-50 \%$. The ratio $\frac{S_{\mathrm{N}, U C D}}{S_{\mathrm{N}, \mathrm{GC}}}$ is $1.00 \pm 0.44$ for the four environments Fornax, Hydra, Centaurus, and Local Group, which have $S_{\mathrm{N}, \mathrm{GC}}$ values. This good match also holds for individual giant galaxies in Fornax and in the Fornax intracluster-space. The error ranges of the derived UCD specific frequencies in the various environments then imply that not more than $\sim 50 \%$ of UCDs were formed from dwarf galaxies. We show that such a scenario would require $\gtrsim 90 \%$ of primordial dwarfs in galaxy cluster centers $(<100 \mathrm{kpc})$ to have been stripped of their stars.

Conclusions. We conclude that the number counts of UCDs are fully consistent with them being the bright tail of the GC population. From a statistical point of view there is no need to invoke an additional formation channel.
\end{abstract}

Key words. galaxies: clusters: general - galaxies: nuclei - galaxies: dwarf - galaxies: star clusters: general - galaxies: star formation

\section{Introduction}

Ultra-compact dwarf galaxies (UCDs) were recognized as a populous and potentially distinct class of objects about a decade ago (Drinkwater et al. 2003), following the results of various spectroscopic surveys in the Fornax cluster (Minniti et al. 1998; Hilker et al. 1999a; Drinkwater et al. 2000; Phillipps et al. 2001). UCDs are generally considered as compact stellar systems with masses above $\simeq 2 \times 10^{6} M_{\odot}$ and sizes below $\sim 100 \mathrm{pc}$ (e.g. Haşegan et al. 2005; Mieske et al. 2008). Since their discovery in Fornax, more UCDs have been detected in a range of environments, from loose and compact galaxy groups (e.g. Evstigneeva et al. 2007; da Rocha et al. 2011) to dense galaxy clusters like Virgo, Centaurus, Hydra, Coma and Fornax itself (e.g. Jones et al. 2006; Haşegan et al. 2005; Misgeld et al. 2008, 2011; Mieske et al. 2004, 2007; Gregg et al. 2009; Chiboucas et al. 2010a,b).

The luminosity and size distribution of UCDs shows a smooth transition to the regime of globular clusters (GCs) (e.g. Haşegan et al. 2005; Mieske et al. 2004, 2006). It is thus not surprising that one of the two discussed formation channels of UCDs is that of massive star clusters created in the same or a similar way to the main star cluster population of early-type galaxies (e.g. Fellhauer \& Kroupa 2002, 2005; Murray 2009; Gieles et al. 2010).

The other proposed formation channel is that of tidally stripped dwarf galaxies (e.g. Zinnecker et al. 1988; Drinkwater et al. 2003; Bekki et al. 2003; Goerdt et al. 2008), as has also been frequently suggested for the Local Group object $\omega$ Cen (e.g. Majewski et al. 2000; Carraro \& Lia 2000; Hilker \& Richtler 2000; Bekki \& Freeman 2003; Noyola et al. 2008; da Costa \& Coleman 2008). Using the mass limit above for the definition of a UCD, indeed $\omega$ Cen is the only UCD associated with the Milky Way (see e.g. the Milky Way GC catalog of Harris et al. 1996).

In this paper we test whether the numbers of UCDs are consistent with being merely the bright $(\sim 1 \%)$ tail of the globular cluster luminosity function (GCLF). A clear excess of UCDs above the canonical GCLF would suggest that a separate process, distinct from the GC formation process, is responsible for the overabundance of UCDs. To this end we define the specific frequency of UCDs, by relating the number of UCDs to the luminosity $M_{V, \text { host }}$ of their host galaxy. The zero point of the definition is chosen such that the UCD specific frequency is equal to that of GCs for the case that the numbers of UCDs are equal to a simple extrapolation of the GCLF. We calculate this specific frequency 
in a range of environments, and compare it to that of star clusters and potential progenitor dwarf galaxies. Special attention is given to the Fornax galaxy cluster, where a large database of confirmed UCDs and GCs exists.

\section{Definition of specific frequency of UCDs}

In this section we define the specific frequency $S_{\mathrm{N}, \mathrm{UCD}}$ of UCDs. Following e.g. Haşegan et al. (2005); Dabringhausen et al. (2008); and Mieske et al. (2008) we define UCDs as compact stellar systems with dynamical masses above $2 \times 10^{6} M_{\odot}$. To convert this to an absolute magnitude limit, we assume an optical mass-to-light ratio $M / L_{V}$ of 2 , which is the average $M / L_{V}$ of compact stellar systems at this limiting mass (Fig. 12 of Mieske et al. 2008) ${ }^{1}$. This therefore yields an absolute limiting magnitude between GCs and UCDs of $M_{V}=-10.25 \mathrm{mag}$, assuming that the Sun's absolute magnitude is $M_{V, \odot}=4.75 \mathrm{mag}$.

One formation scenario of UCDs is that they constitute the high-mass tail of the star cluster population (e.g. Hilker et al. 1999a; Drinkwater et al. 2000; Fellhauer \& Kroupa 2002; Mieske et al. 2002, 2004; Murray 2009; Gieles et al. 2010; Frank et al. 2011). Therefore, our aim is a quantitative comparison of the specific frequency of UCDs to those of GCs. To calculate the UCD specific frequency we relate the number of UCDs to the luminosity $M_{V \text {,host }}$ of its host galaxy, analogous to the case of GCs (Harris \& van den Bergh 1981). The prerequisite in that context is that the specific frequency of UCDs should be equal to that of GCs if the luminosity distribution of UCDs is consistent with a simple extrapolation of the GCLF to brighter magnitudes. This is illustrated in Fig. 1 for the default assumption of a Gaussian $\operatorname{GCLF} N\left(M_{V}\right) \propto e^{\frac{-\left(M_{V, \mathrm{TOM}}-M_{V}\right)^{2}}{2 \sigma^{2}}}$.

The term $M_{V, \text { TOM }}$ denotes the turnover-magnitude (=TOM) of the GCLF, which is the maximum of the log-normal luminosity function. The term $\sigma$ denotes the characteristic width of that GCLF, which has typical values between 1.0 and 1.4 mag. For the UCD specific frequency we choose the same functional form that is used for the specific frequency of GCs (Harris \& van den Bergh 1981; Peng et al. 2008, and references therein).

$S_{\mathrm{N}, \mathrm{UCD}}=N_{\mathrm{UCD}} 10^{0.4\left(M_{V, \text { host }}-M_{V, 0}\right)} c_{\mathrm{W}}$.

For $c_{\mathrm{w}}=1, M_{V, 0}$ is the galaxy luminosity at which for a specific frequency $S_{\mathrm{N}, \mathrm{UCD}}=1$ one would expect exactly one UCD. Given the premise to make $S_{\mathrm{N}, \mathrm{UCD}}$ directly comparable to $S_{\mathrm{N}, \mathrm{GC}}$, the value of $M_{V, 0}$ depends on which fraction of the area below the canonical GCLF is occupied by the UCD luminosity range. The correction term $c_{\mathrm{w}}$ is included to account for the varying width of the GCLF as a function of host galaxy magnitude (e.g. Jordán et al. 2007). If the GCLF of the investigated host galaxy is identical to the reference GCLF (see below), $c_{\mathrm{w}}=1$. The exact functional form of $c_{\mathrm{w}}$ as a function of GCLF width is discussed at the end of this Section. For convenience in the further course of the paper, we also introduce the "reference" specific frequency $S_{\mathrm{N}, \mathrm{UCD}}^{*}=S_{\mathrm{N}, \mathrm{UCD}} / c_{\mathrm{w}}$, which is the frequency for when all galaxies have the same (reference) GCLF width.

We furthermore define the quantity $n_{\mathrm{UCD}}$ as the fraction of sources below a normalized Gaussian GCLF that have luminosities $M_{V}<-10.25 \mathrm{mag}$, the UCD luminosity limit. For $M_{V, 0}$ it then holds that

$M_{V, 0}=-15+2.5 \log n_{\mathrm{UCD}}$.

\footnotetext{
${ }^{1}$ For higher masses and metallicities, $M / L_{V}$ increases to larger average values of 4-5 (Mieske et al. 2008).
}

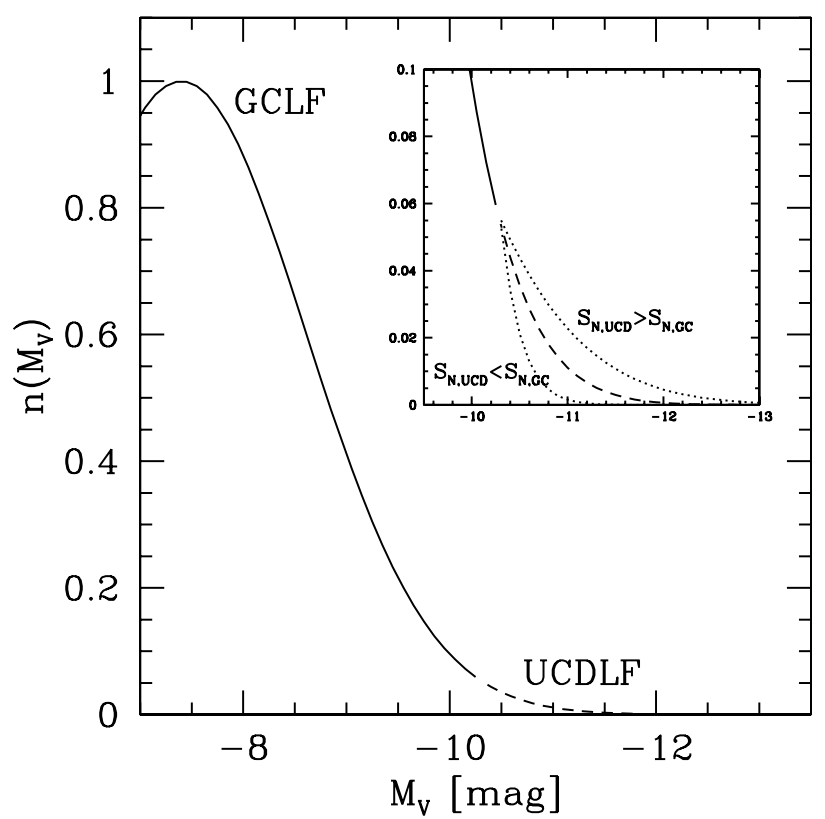

Fig. 1. This plot illustrates the definition of the UCD specific frequency $S_{\mathrm{N}, \mathrm{UCD}}$. The solid line represents a typical GC luminosity function (GCLF) in the shape of a Gaussian, centered at $M_{V}=-7.4 \mathrm{mag}$, with a width of $\sigma=1.2 \mathrm{mag}$. The line becomes dashed in the magnitude range of UCDs $M_{V}<-10.25$ mag. It holds that $S_{\mathrm{N}, \mathrm{UCD}}=S_{\mathrm{N}, \mathrm{GC}}$ if the luminosity distribution of UCDs follows the extrapolated GCLFs, and that $S_{\mathrm{N}, \mathrm{UCD}}>S_{\mathrm{N}, \mathrm{GC}}$ if the frequency of UCDs is above the GCLF extrapolation (upper dotted line in inset), and $S_{\mathrm{N}, \mathrm{UCD}}<S_{\mathrm{N}, \mathrm{GC}}$ if it is below (lower dotted line in inset).

The term -15 comes directly from the definition of the GC specific frequency, for which $M_{V, 0}=-15 \mathrm{mag}$ (Harris \& van den Bergh 1981). In the following we consider a Gaussian GCLF with a turnover-magnitude of $M_{V, \text { TOM }}=-7.4$ mag (e.g. Harris 1991; Kundu \& Whitmore 2001; Jordán et al. 2006; Peng et al. 2008), see also Fig. 1. The width of the GCLF is typically $\sigma=1.2 \mathrm{mag}$ for galaxies with absolute magnitudes of around $M_{V}=-22 \mathrm{mag}$. As reference width we adopt $\sigma=$ $1.223 \mathrm{mag}$, since this corresponds exactly to the case where $1 \%$ of the area below the GCLF falls into the UCD magnitude range $M_{V}<-10.25 \mathrm{mag}$, hence $n_{\mathrm{UCD}}=0.01$. With this reference we obtain a "simple" value of $M_{V, 0}=-20 \mathrm{mag}$, five magnitudes offset from the zero point of the GC specific frequency. For a UCD specific frequency of unity in the case of the Milky Way $\left(M_{V}=-20.5 \mathrm{mag}\right)$, one would thus expect 1-2 UCDs, which corresponds to reality since $\omega$ Cen is the only MW satellite that satisfies the UCD definition. At the same time, a specific frequency around unity is also typically found for the GC populations of Milky Way type galaxies (e.g. Goudfrooij et al. 2003; Rhode \& Zepf 2004; Chandar et al. 2004). Given the prerequisites we imposed for the definition of $S_{\mathrm{N}, \mathrm{UCD}}$, this shows that the number of UCDs in the Milky Way is about consistent with what is expected from an extrapolation of its GCLF. In the next section this is discussed in more detail for a range of environments.

While for the Milky Way and Local Group the luminosity distribution of compact stellar systems is sampled down to very faint absolute magnitudes well into the regime of bona-fide GCs, this is not necessarily the case for most galaxy clusters where UCDs have been found (Drinkwater et al. 2000; Phillipps et al. 2001; Mieske et al. 2004, 2007; Misgeld et al. 2011), at distance moduli in the range $31<(m-M)<33.5 \mathrm{mag}$, and even $35 \mathrm{mag}$ for Coma (Chiboucas et al. 2010a,b). The 
Table 1. List of the specific frequencies $S_{\mathrm{N}, \mathrm{UCD}}$ derived in Sect. 3, along with the total absolute magnitude of the host environments.

\begin{tabular}{lccccc}
\hline \hline Environment & $S_{\mathrm{N}, \mathrm{UCD}}$ & $M_{V \text {,host }}$ & $M_{V \text {,host,individual }}$ & $c_{\mathrm{W}}$ & $S_{\mathrm{N}, \mathrm{GC}}$ \\
\hline Centaurus & $5.1 \pm 1.7$ & -23.2 & -23.2 & 0.38 & $7.3 \pm 1.5^{*}$ \\
Fornax & $4.9 \pm 1.7$ & -23.0 & -22.6 & 0.54 & $5.1 \pm 1.2^{* *}$ \\
Hydra & $12.5 \pm 4.3$ & -22.9 & -22.5 & 0.58 & $12.5 \pm 1.5^{* * *}$ \\
HCG 90 & $1.3 \pm 0.7$ & -22.4 & -21.1 & 1.41 & \\
HCG 22 & $3.9 \pm 1.6$ & -21.6 & -21.1 & 1.41 & \\
Local Group & $4.1 \pm 1.5$ & -21.5 & -21.0 & 1.5 & $1.8 \pm 0.3^{* * * *}$ \\
\hline
\end{tabular}

Notes. $M_{V \text {,host }}$ includes the full galaxy light in the UCD survey area (see text). $M_{V \text {,host,individual }}$ gives the magnitude of the brightest galaxy in the environment, which defines the assumed width $\sigma$ of the GCLF and hence the correction term $c_{\mathrm{w}}$. The last column gives the literature value for the GC specific frequency of the brightest cluster galaxy, where available.

References. ${ }^{(*)}$ Mieske et al. (2005); ${ }^{(* *)}$ Dirsch et al. (2003); ${ }^{(* * *)}$ Wehner et al. (2008); ${ }^{(* * *)}$ Barmby \& Huchra (2001); Gil de Paz. (2007).

completeness of the surveys within $(m-M)<33.5$ mag in terms of slit allocation, area coverage, and spectroscopic success rate is well defined and around $50 \%$ for $M_{V} \lesssim-11$ mag. However, at fainter magnitudes the available survey data are very heterogeneous in terms of target selection, slit allocation, area selection, and spectroscopic "success rate" (see references above). This needs to be accounted for in the calculation of the specific frequency of UCDs. We therefore define an equivalent formulation of $S_{\mathrm{N}, \mathrm{UCD}}$ for the case that the UCD sample is only well known for $M_{V}<-11 \mathrm{mag}$. In the context of the $S_{\mathrm{N}, \mathrm{UCD}}$ definition above, the change we need to adopt for restricting the UCD sample to $M_{V}<-11 \mathrm{mag}$ is the value of $M_{V, 0}$. The limit of $M_{V}<-11 \mathrm{mag}$ corresponds to $2.95 \sigma$ away from the turnover magnitude for a reference GCLF width $\sigma=1.223$ mag, yielding a fraction of 0.00159 of all sources. This is 6.29 times less than for the default limit of $M_{V}<-10.25 \mathrm{mag}$, or quite exactly $2.0 \mathrm{mag}$. Therefore, we adopt $M_{V, 0}=-22 \mathrm{mag}$ when the UCD sample is restricted to $M_{V}<-11 \mathrm{mag}$. The two formulations with different $M_{V, 0}$ are considered equivalent in the context of our study, where we want to test the consistency of the UCD luminosity distribution with the extrapolation of the GCLF.

\subsection{The correction term $c_{w}$}

The fractional area $A$ below a Gaussian GCLF brighter than a given magnitude $M_{V}$ is related to the error function $\operatorname{erf}$ in the following way

$A=1-0.5\left(1+\operatorname{erf}\left(\frac{M_{V}+7.4}{\sigma \sqrt{2}}\right)\right)$.

From this and the reference of $\sigma=1.223$, it follows for the definition of $c_{\mathrm{w}}$ for a fully sampled UCDLF down to $M_{V}<-10.25$ mag that

$c_{\mathrm{W}}=\frac{1-0.5\left(1+\operatorname{erf}\left(\frac{2.85}{\sigma \sqrt{2}}\right)\right)}{1-0.5\left(1+\operatorname{erf}\left(\frac{2.85}{1.223 \sqrt{2}}\right)\right)}$.

For a partially sampled UCDLF down to $M_{V}<-11$ mag the following then holds:

$c_{\mathrm{W}}=\frac{1-0.5\left(1+\operatorname{erf}\left(\frac{3.6}{\sigma \sqrt{2}}\right)\right)}{1-0.5\left(1+\operatorname{erf}\left(\frac{3.6}{1.223 \sqrt{2}}\right)\right)}$.

It is well known that the width $\sigma$ of the GCLF depends on the host galaxy luminosity $M_{V \text {,host,individual, becoming higher for }}$ brighter host galaxies (e.g. Jordán et al. 2007). To take this into account for the correct determination of $c_{\mathrm{w}}$, we adapt Eq. (18) of Jordán et al. (2007) to calculate $\sigma$ as a function of $M_{V \text {,host,individual }}$ :

$\sigma=1.14-0.100\left(M_{V, \text { host,individual }}+20.9\right)$.

For this we have explicitly assumed $(B-V)=0.9$ mag, which is the color expected for a $\sim 10 \mathrm{Gyr}$ single-burst stellar population of $[\mathrm{Fe} / \mathrm{H}] \sim-0.7$ dex (Bruzual \& Charlot 2003). We furthermore assume that the GCLF width in the SDSS g-band (Jordán et al. 2007) is identical to the width in the $V$-band. It is important to note that the term $M_{V, \text { host,individual }}$ refers to the luminosity of an individual host galaxy. For the calculation of the specific frequency, we need to normalize the number of UCDs to the total galaxy luminosity in the surveyed area, which can be some 0.3-0.5 mag brighter than the luminosity of the brightest individual galaxy (see next section).

\section{Specific frequency as a function of environment}

In this section we calculate the specific frequency of UCDs in the three massive nearby galaxy clusters Fornax, Centaurus, and Hydra, based mainly on data of spectroscopic surveys performed within our group, except for Fornax for which a wealth of datasets is available. The projected spatial distribution of UCDs in those surveys is shown in Fig. 2. We do not include the recent data from the Coma cluster (Chiboucas et al. 2010a) due to its greater distance, larger incompleteness in terms of magnitude coverage, and complex selection function of UCD candidates. Furthermore we calculate the UCD specific frequency for the Local Group, and two compact galaxy groups recently investigated in Da Rocha et al. (2011). The results of these calculations are summarized in Table 1 and Fig. 6, in which we also compare the derived UCD specific frequencies with the frequency of GCs.

\subsection{Fornax}

In this section we analyze the specific frequencies of UCDs in the Fornax cluster. We start with an analysis of the very central Fornax part, based on our UCD survey from Mieske et al. (2004), for which the survey completeness is quantified well and which extends down to $M_{V} \simeq-10.4 \mathrm{mag}$. Then we extend the analysis to the entire cluster by including all available literature results. This includes also the earlier Fornax survey performed with the $2 \mathrm{dF}$ spectrograph (e.g. Drinkwater et al. 2000), which had a complete areal coverage in the central cluster, but a significantly brighter magnitude limit $M_{V} \lesssim-12 \mathrm{mag}$. Assumed distance modulus of Fornax is 31.4 mag (Ferrarese et al. 2000). 

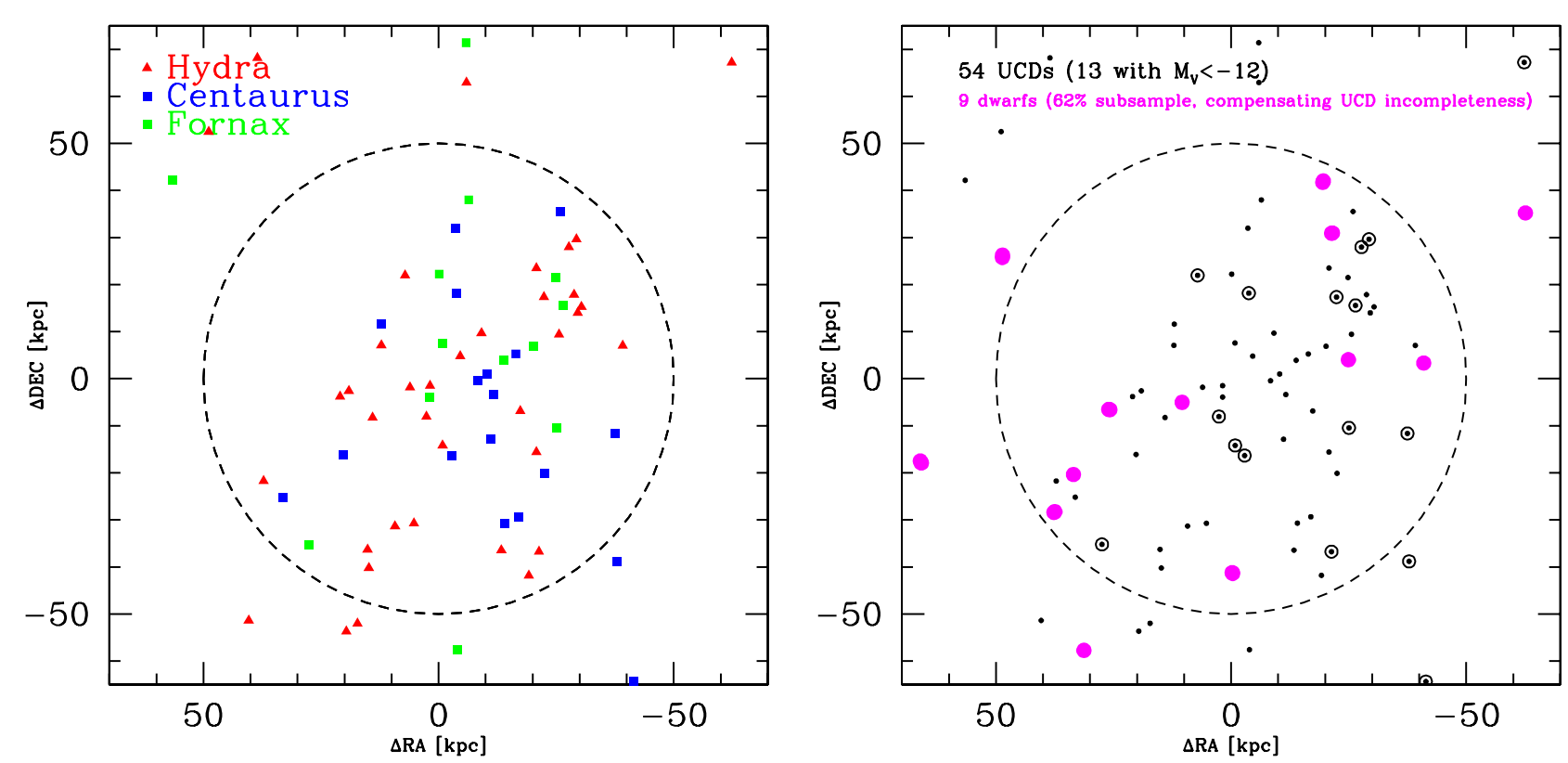

Fig. 2. Projected spatial distribution of UCDs within the inner $\simeq 100 \mathrm{kpc}$ of the Hydra, Centaurus and Fornax clusters (Misgeld et al. 2009, 2011; Mieske et al. 2004). UCDs are in this context defined as compact stellar systems with $M_{V}<-11$ mag. Left panel: UCDs color coded according to host cluster. Red triangles are Hydra UCDs, blue squares are Centaurus UCDs, and green circles are Fornax UCDs. Right panel: the same sample of UCDs is shown as black dots, with the brightest UCDs $\left(M_{V}<-12 \mathrm{mag}\right)$ marked by large open circles. There are 54 UCDs known within the central $50 \mathrm{kpc}$ of Hydra, Centaurus, and Fornax. Large filled circles indicate the projected positions of canonical dwarf galaxies (see text for references), restricted to a magnitude range $-20.5<M_{V}<-16 \mathrm{mag}$, which corresponds to the assumed luminosity range for possible UCD progenitor galaxies (see text for details). To allow a direct comparison of the number counts between UCDs and progenitors, we show only $62 \%$ of the dwarf galaxies, given that the UCD searches are on average complete to $\sim 62 \%$ in the central $50 \mathrm{kpc}$ radius (see text for details).

\subsubsection{The central region}

In Mieske et al. (2004) we describe our search for compact stellar systems in the central $\sim 100 \mathrm{kpc}$ radius ( $20 \mathrm{arcmin})$ of the Fornax cluster, which was performed with the WFCCD camera at the $2.5 \mathrm{~m}$ du Pont telescope at Las Campanas Observatory. In Fig. 2 we show the location of spectroscopically confirmed UCDs with $M_{V}<-11 \mathrm{mag}$, restricted to the inner 70-80 kpc around NGC 1399. Some of these UCDs are outside of the plot. In Mieske et al. (2004), we calculated the overall survey completeness for $M_{V}<-11 \mathrm{mag}$ to be $70 \% \pm 5 \%$ in our survey area, which comprises the two bright gE galaxies NGC 1399 and NGC 1404. This number takes the varying radial density of confirmed cluster members compared to background sources into account. The completeness in terms of slit allocation and spectroscopic success is calculated in rings, and the global value of the completeness is the weighted mean of those values, weighted by the number of UCDs found in each ring.

There are 20 confirmed UCDs with $M_{V}<-11$ mag in our survey. Given the global completeness of $70 \%$, this translates into a total number of $28.6 \pm 7$ UCDs with $M_{V}<-11$ mag in the central Fornax cluster. In the surveyed region the two gE galaxies NGC 1399 and NGC 1404 contribute the lions share of the galaxy light. NGC 1399 has $M_{V}=-22.6$ mag within $\sim 10^{\prime}$ radius ( $\simeq 55 \mathrm{kpc}$; Dirsch et al. 2003), while NGC 1404 is about $1 \mathrm{mag}$ fainter (NED). The sum of both absolute magnitudes therefore corresponds to $M_{V} \simeq-23.0 \mathrm{mag}$. We assume an uncertainty of 0.2 mag for this absolute magnitude based on experience with light profile fitting and the related uncertainty in the accurate determination of the sky background. We thus obtain a reference specific frequency of $S_{\mathrm{N}, \mathrm{UCD}}^{*}=11.4 \pm 4$ for the central $100 \mathrm{kpc}$ of Fornax. If we restrict this calculation to the inner $\sim 10^{\prime}$ (=55 kpc), we can disregard the contribution of NGC 1404 , but at the same time only have 12 UCDs with $M_{V}<-11$ mag. This would yield a lower specific frequency of $S_{\mathrm{N}, \mathrm{UCD}}^{*} \simeq 7$. We adopt the mean of both estimates $S_{\mathrm{N}, \mathrm{UCD}} * \simeq \pm 3$. Evaluating $c_{\mathrm{W}}$ for $M_{V}=-22.6 \mathrm{mag}$ (the luminosity of the gE NGC 1399, which dominates in terms of associated GCs) then yields a final value of $S_{\mathrm{N}, \mathrm{UCD}}=4.9 \pm 1.7$. This downward correction is because the GCLF width $\sigma$ for $M_{V}=-22.6$ mag is 1.31 mag according to Eq. (6), which implies almost twice as many sources in the bright end tail $M_{V}<-11$ mag than for the reference width of $\sigma=1.223 \mathrm{mag}$. For comparison, the GC specific frequency of NGC 1399 is $S_{\mathrm{N}, \mathrm{GC}}=5.1 \pm 1.2$ (Dirsch et al. 2003), fully consistent with the value for UCDs.

\subsubsection{The overall cluster}

Restricting our considerations in the previous section to the very central Fornax cluster with only one survey has the advantage of a homogeneous catalog and of well controlled completeness. However, for the Fornax cluster there is an extraordinarily large database of further spectroscopcically confirmed compact cluster members, which is worth analyzing in the context of the present study. The caveat is that this database is heterogeneous in survey design and coverage.

In Fig. 3 we show the distribution of all compact objects (UCDs + GCs) in the Fornax cluster (adopted from Fig. 1 of Hilker 2011). Their magnitude distribution is shown in Fig. 4. The spectroscopy and photometry has been compiled from Kissler-Patig et al. (1999), Drinkwater et al. (2000), Dirsch et al. (2003), Mieske et al. (2004), Bassino et al. (2006a), Bergond et al. (2007), Firth et al. (2007, 2008), Jordán et al. (2007), Schuberth et al. (2010), Gregg et al. (2009), Puzia et al. (2011 priv. comm.). Small black dots are GCs 


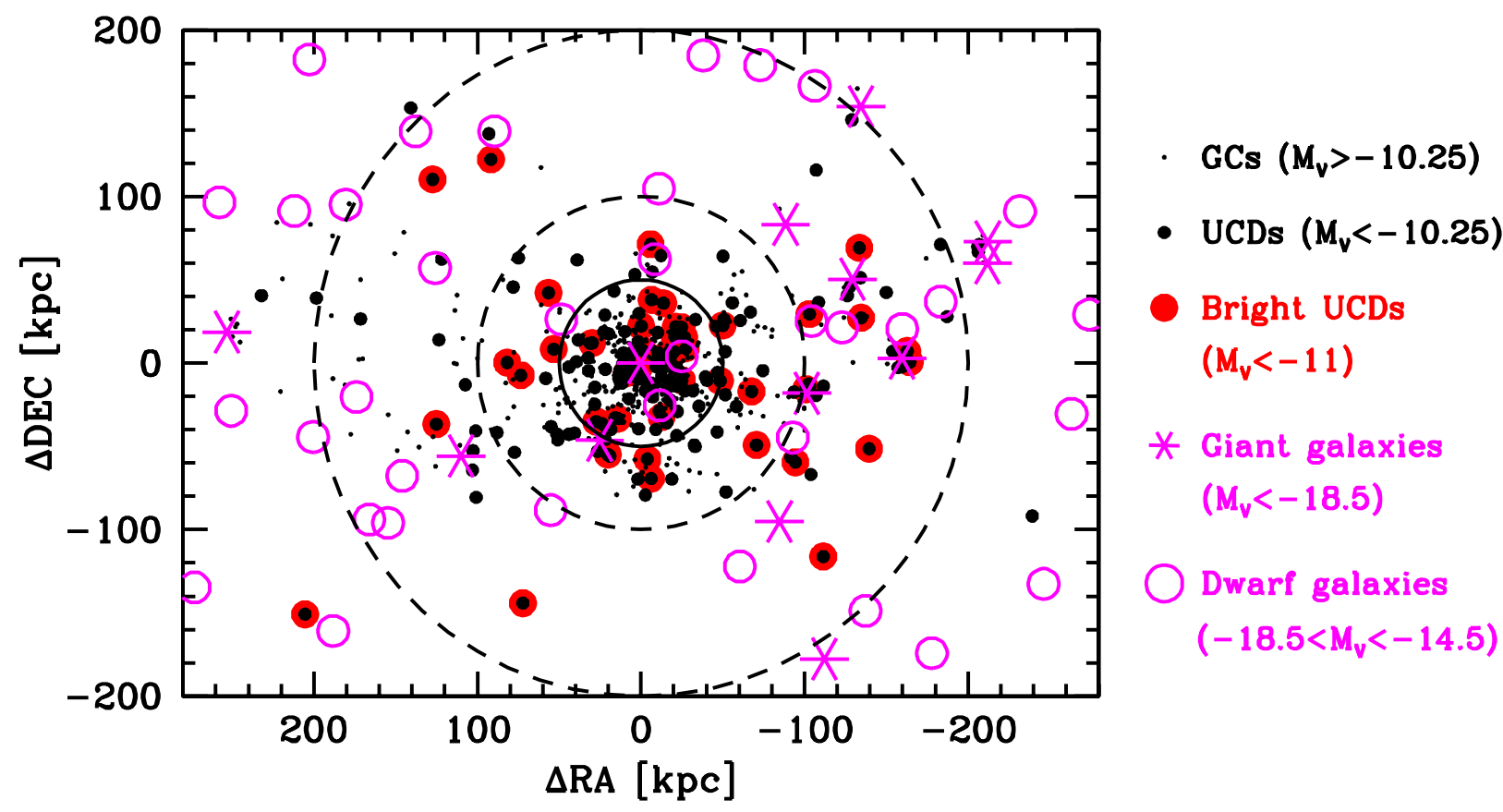

Fig. 3. Projected distribution of all confirmed UCDs and GCs in the Fornax cluster (see text; spectroscopy and photometry compiled from Kissler-Patig et al. 1999; Drinkwater et al. 2000; Dirsch et al. 2003; Mieske et al. 2004; Bassino et al. 2006a; Bergond et al. 2007; Firth et al. 2007, 2008; Jordán et al. 2007; Schuberth et al. 2010; Gregg et al. 2009; Puzia et al. 2011, priv. comm.). Small black dots are GCs $\left(M_{V}>-10.25\right.$ mag; about 400 sources). Filled black circles are UCDs $\left(M_{V}<-10.25\right.$ mag; about 180 sources). Filled red circles are "bright" UCDs with $M_{V}<-11$ mag, about 45 sources. Large magenta asterisks indicate Fornax cluster giant galaxies from the FCC, defined as having $M_{V}<-18.5$ mag. Magenta open circles indicate dwarf galaxies from the FCC with $-18.5<M_{V}<-14$ mag, the postulated approximate magnitude range of UCD progenitor galaxies (Bekki et al. 2003). The inner solid circle indicates a radius of $50 \mathrm{kpc}$ adopted in Fig. 2 for the comparison between the Fornax, Hydra, and Centaurus environments. As can be seen, the area coverage of the available Fornax spectroscopic surveys drops strongly beyond $50 \mathrm{kpc}$ radius.

$\left(M_{V}>-10.25 \mathrm{mag}\right.$; about 400 sources $)$. Filled black circles are UCDs $\left(M_{V}<-10.25 \mathrm{mag}\right.$; about 180 sources). Filled red circles are "bright" UCDs with $M_{V}<-11 \mathrm{mag}$, about 45 sources. Large magenta asterisks indicate Fornax cluster giant galaxies from the FCC (Fornax Cluster Catalogue; Ferguson \& Sandage 1988), defined as having $M_{V}<-18.5 \mathrm{mag}$. Magenta open circles indicate dwarf galaxies from the FCC with $-18.5<M_{V}<-14$ mag, the postulated approximate magnitude range of UCD progenitor galaxies (Bekki et al. 2003) ${ }^{2}$. The inner solid circle indicates the radius of $50 \mathrm{kpc}$, which is adopted for the comparative analysis of the Fornax, Hydra and Centaurus environments (see previous and next sections).

The area coverage of the available Fornax spectroscopic surveys starts to become patchy beyond $50 \mathrm{kpc}$. Many detections between 100 and $200 \mathrm{kpc}$ clustercentric distance are from the VLT/FLAMES survey of Bergond et al. (2007), which focuses on a strip of $500 \times 150 \mathrm{kpc}$ aligned along the east-west axis to cover the giant galaxy distribution in the central Fornax cluster. Another important contribution comes from the VLT/FLAMES survey of Firth et al. (2007) in the inner $130 \mathrm{kpc}$ radius of Fornax. These authors quote a spatial completeness of 15-30\% between 80 and $180 \mathrm{kpc}$ radius. Overall, from these literature sources and Fig. 3 we estimate a spatial coverage of $60-70 \%$ between 50 and $100 \mathrm{kpc}$ and $30-50 \%$ beyond $100 \mathrm{kpc}$. Based on the data from Fig. 3 we address four aspects that go beyond the focus of the previous subsection:

1. What is the specific frequency of UCDs in the inner $50 \mathrm{kpc}$ around NGC 1399, including all literature detections down

\footnotetext{
${ }^{2}$ See also discussion on the progenitor magnitude range in Sect. 4.
}

to $M_{V}<-10.25 \mathrm{mag}$ ? The data shown in Fig. 3 give a total number of 84 spectroscopically confirmed UCDs with $M_{V}<-10.25 \mathrm{mag}$, and $20 \mathrm{UCDs}$ with $M_{V}<-11 \mathrm{mag}$ in the inner $50 \mathrm{kpc}$. The latter number is consistent with the completeness correction of the survey in Mieske et al. (2004), which would predict $\sim 17$ UCDs with $M_{V}<-11$ mag in the inner $50 \mathrm{kpc}$. Also, the magnitude distribution of confirmed UCDs \& GCs (Fig. 4) is fully consistent with the GCLF shape for $M_{V} \lesssim-10.5 \mathrm{mag}$, while the spectroscopic incompleteness becomes notable for $M_{V} \gtrsim-10 \mathrm{mag}$. For the innermost $50 \mathrm{kpc}$, we therefore use the working hypothesis of a complete sample down to the UCD limit of $M_{V}=-10.25 \mathrm{mag}$.

We thus find $S_{\mathrm{N}, \mathrm{UCD}}=6.2 \pm 2.4$ for considering UCDs with $M_{V}<-11 \mathrm{mag}$, and $S_{\mathrm{N}, \mathrm{UCD}}=4.1 \pm 0.5$ for considering UCDs with $M_{V}<-10.25 \mathrm{mag}$. Both values agree with the result derived from the UCD sample of Mieske et al. (2004) alone and with the specific frequency of GCs in NGC 1399 (Dirsch et al. 2003).

2. How does the number of UCDs associated to individual galaxies outside of $50 \mathrm{kpc}$ compare to the prediction assuming $S_{\mathrm{N}, \mathrm{UCD}}=3$ ? The particular choice of $S_{\mathrm{N}, \mathrm{UCD}}=3$ is to represent typical GC specific frequencies in the Fornax cluster giant galaxies, which range from 1.5 to 5 (Dirsch et al. 2003; Bassino et al. 2006b; Kissler-Patig et al. 1997). Integrating for $S_{\mathrm{N}, \mathrm{UCD}}=3$ over all nine giant galaxies beyond NGC 1399/1404 which fall into UCD survey regions, one would expect a total of 18 UCDs associated to those galaxies. To test this, we restricted to UCDs within $20 \mathrm{kpc}$ projected distance from the galaxies. For the Milky Way, this 


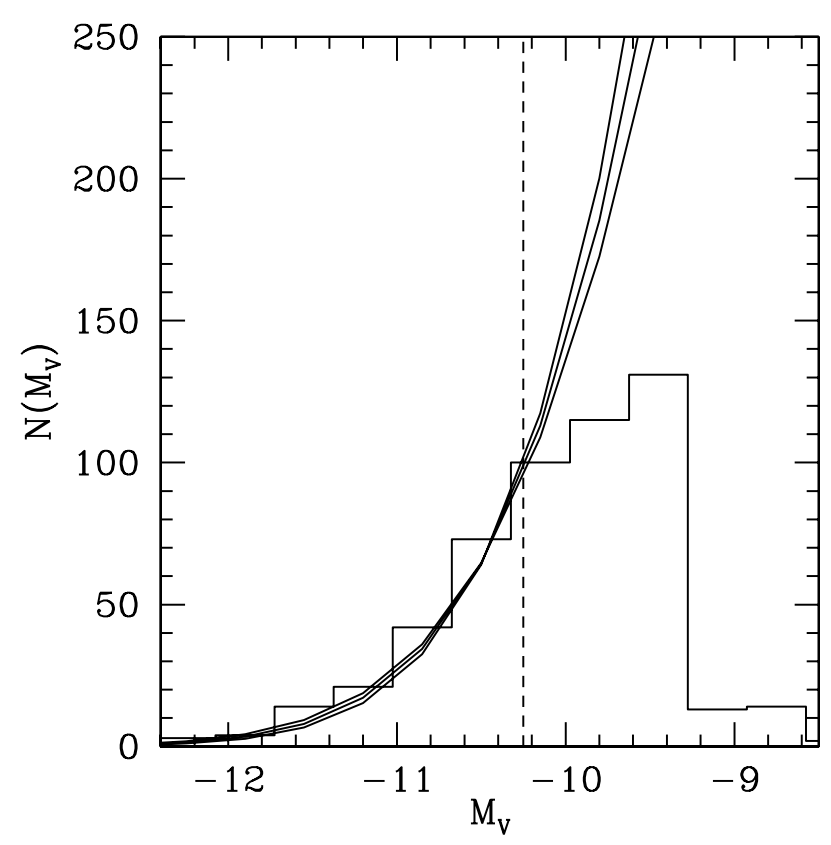

Fig. 4. Absolute magnitude distribution of GCs and UCDs shown in Fig. 3. The dotted vertical line indicates the magnitude limit adopted between UCDs and GCs. The curves show Gaussian GCLFs normalized to match the object counts at the UCD magnitude limit. The three lines correspond to different widths $\sigma$ of the Gaussians: 1.30, 1.35, and $1.40 \mathrm{mag}$. The completeness of the spectroscopic surveys drops at about the UCD limit.

restriction would encompass 85-90\% of GCs (Harris 1996). From the data in Fig. 3 we then find a total of 25 UCDs associated to the nine giant galaxies. This number is consistent with the $18 \mathrm{UCDs}$ predicted for $S_{\mathrm{N}, \mathrm{UCD}}=3$. Given the possible incompleteness in the target selection and spectroscopic success rates of the various surveys, these are lower limits to the number of existing UCDs.

3. How does the number of spectroscopically confirmed UCDs in the intracluster region (100-200 kpc distance) compare to the GC surface density determined statistically from photometry (Bassino et al. 2006a)? From Fig. 3 it is clear that many UCDs and GCs are found in regions that are not directly associated to any giant galaxy. Between a clustercentric radius of 100 and $200 \mathrm{kpc}$ we find a total of 40 UCDs with $M_{V}<-10.25 \mathrm{mag}$, and about ten UCDs with $M_{V}<$ $-11 \mathrm{mag}$. Due to the low number counts we focus on UCDs with $M_{V}<-10.25 \mathrm{mag}$. Of those, 25 are associated to giant galaxies (see previous item). We are thus left with a sample of $\sim 15 \pm 5$ UCDs in the intracluster space between 100 and $200 \mathrm{kpc}$. In the ring between $100 \mathrm{kpc}$ and $200 \mathrm{kpc}$, we expect about 1000-1100 GCs according to the photometrically estimated surface density of GCs in that region measured by Bassino et al. (2006a), Table 2. For a GCLF width of $\sigma=1.31 \mathrm{mag}$ for NGC 1399 (see previous subsection), we would thus expect $\sim 20$ sources in the UCD magnitude regime $M_{V}<-10.25 \mathrm{mag}$. This matches the confirmed number of $\sim 15$ intracluster UCDs well. However the latter value is a lower limit to the true number of UCDs given that only about one third of the area between $100 \mathrm{kpc}$ and $200 \mathrm{kpc}$ has been surveyed spectroscopically (Fig. 3 and text above). A complete spectroscopic survey in this region may still lead to an overabundance of UCDs with respect to the IC GCLF of up to a factor of $\sim 2$.
4. Is there any evidence for different spatial distributions of UCDs and GCs? In Fig. 5 we show the projected radial distribution of spectroscopically confirmed UCDs and GCs from Fig. 3. GCs are shown with the dashed lines. UCDs $\left(M_{V}<\right.$ $-10.25 \mathrm{mag}$ ) are shown as solid lines, and bright UCDs $\left(M_{V}<-11 \mathrm{mag}\right)$ as dotted lines. The red curves correspond to the samples restricted to the inner $50 \mathrm{kpc}$. The blue curves correspond to the sample excluding the inner $50 \mathrm{kpc}$. No significant distribution difference between GCs and UCDs is seen for the outer region beyond $50 \mathrm{kpc}$. For the inner region within $50 \mathrm{kpc}$, there is mild evidence of a more extended distribution of bright UCDs $\left(M_{V}<-11 \mathrm{mag}\right)$ compared to GCs. The KS test shows an $8 \%$ probability that both samples are drawn from the same parent distribution. This corroborates a similar finding by Mieske et al. (2004), which shows a $12 \%$ probability that sources with $M_{V}<-11$ mag have the same parent distribution as sources with $M_{V}>-11 \mathrm{mag}$ (see also Fig. 2 of Hilker 2011).

We conclude that the specific frequencies of confirmed UCDs in the Fornax cluster agree with that of GCs to within their errors. Also a comparison of spatial distribution shows only mild differences between UCDs and GCs.

The specific frequencies of UCDs will clearly rise to some extent, once complete spectroscopic surveys down to $M_{V} \sim$ -10 mag are available for the central Mpc of the Fornax cluster. It is, however, difficult to predict the magnitude of this effect, or to predict whether it will give a significant difference between $S_{\mathrm{N}, \mathrm{UCD}}$ and $S_{\mathrm{N}, \mathrm{GC}}$. Based on the rectangular survey coverage (Fig. 3, Bergond et al. 2007) one may speculate that the number of UCDs could still rise by up to a factor of $\sim 2$ in the outer cluster parts. However, this depends on the actual spatial distribution of UCDs in these unsurveyed regions, which is unknown. The overall galaxy distribution is clearly elongated along the survey coverage of Bergond et al. (2007), such that a factor of two increase in UCD number counts from the uncovered regions appears a reasonable upper limit.

\subsection{Centaurus}

For calculating the specific frequencies of UCDs in the Centaurus cluster, we used the data presented in Mieske et al. (2007, 2009), based on two surveys performed with VIMOS@VLT. Figure 2 shows a map of the central Centaurus cluster regions with the same physical plot limits as for Fornax. The assumed distance modulus of Centaurus is $33.28 \mathrm{mag}$ (Mieske et al. 2005). About three quarters of the UCDs in Centaurus were detected in one central VIMOS quadrant around the main galaxy NGC 4696, whose dimension is $7 \times 7^{\prime}$, or $85 \times 85 \mathrm{kpc}$ (see e.g. Fig. 1 of Mieske et al. 2007). We therefore restrict our considerations to this pointing and central quadrant and assume $M_{V \text {,host }}=-23.2 \pm 0.2 \mathrm{mag}$ (Misgeld et al. 2008) for NGC 4696. Figure 2 shows that in Centaurus we detected 18-19 UCDs with $M_{V}<-11$ mag within the central $\sim 50 \mathrm{kpc}$. In the UCD survey from Mieske et al. (2007), within which all but one UCD considered here were detected, we estimate the survey completeness in terms of slit allocation and spectroscopic success to be 0.29 . However, the region under consideration in Fig. 3 was covered twice in that survey, such that the completeness improves to $0.29+(0.29 *(1-0.29))=0.50$, with an estimated uncertainty of 0.07 . The absolute magnitude $M_{V \text {,host }}=-23.2 \pm 0.2$ mag of NGC 4696 is taken from a FORS2 imaging survey (Misgeld et al. 2008) which covers the approximately same pointing, such that we do not have to apply any 

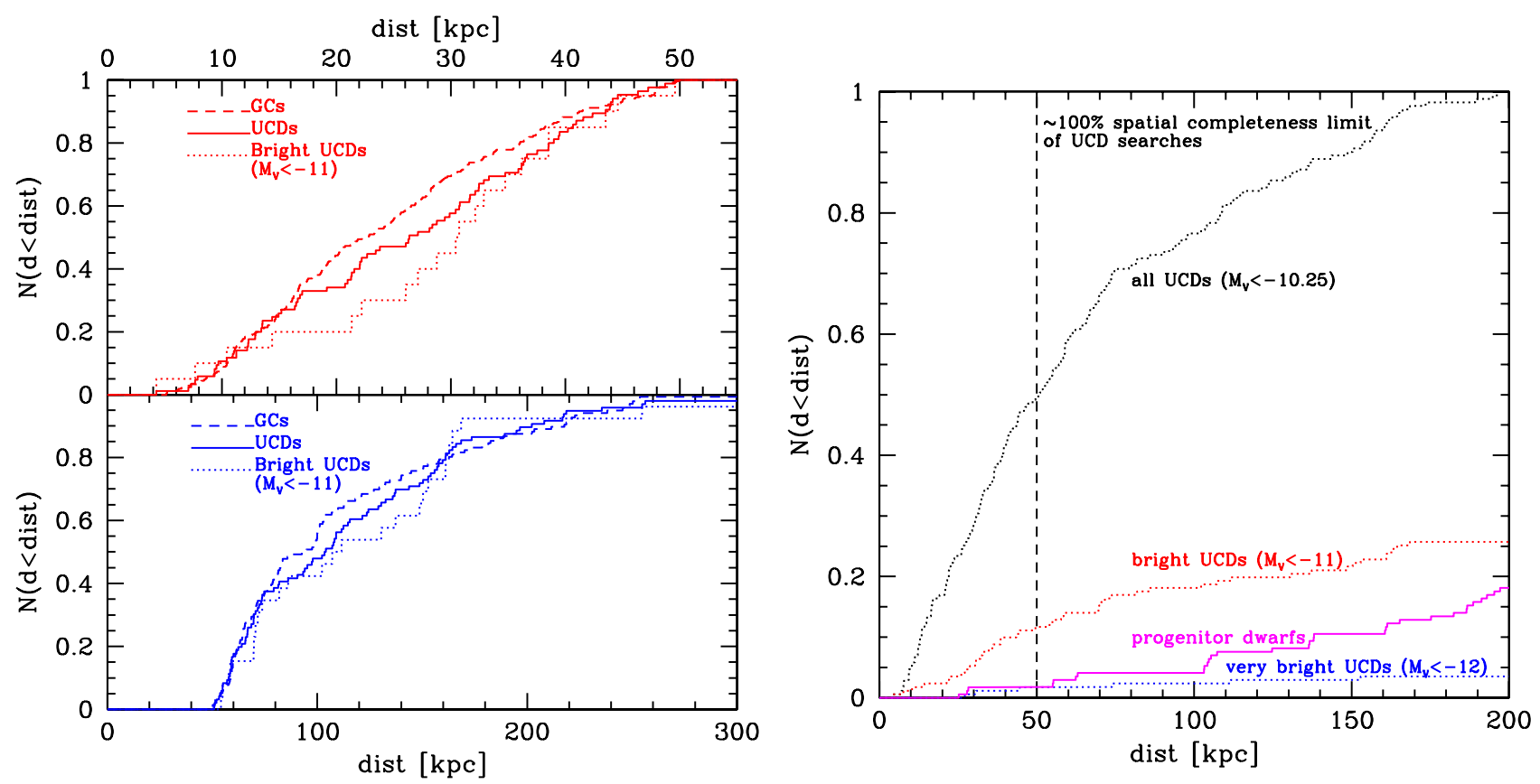

Fig. 5. Left panel: projected radial distribution of GCs and UCDs in the Fornax cluster, see also Fig. 3. The upper plot with the red curves corresponds to the distribution within $50 \mathrm{kpc}$, with $x$-axis limits on the top. The lower plot with the blue curves corresponds to the distribution outside of $50 \mathrm{kpc}$, with $x$-axis limits on the bottom. Solid lines are UCDs $\left(M_{V}<-10.25 \mathrm{mag}\right)$. Dotted lines are bright UCDs with $M_{V}<-11.0 \mathrm{mag}$. Dashed lines are GCs $\left(M_{V}>-10.25 \mathrm{mag}\right)$. There is mild evidence (92\% significance according to a KS test) of a more extended distribution of bright UCDs compared to GCs within the inner $50 \mathrm{kpc}$. Right panel: cumulative radial distribution of UCDs (black, red, and blue curves), compared to that of dwarf galaxies (magenta) in the postulated progenitor magnitude range of $-18.5<M_{V}<-14$ mag (Bekki et al. 2003); see also Fig. 3 . The maximum of the various curves have been re-normalized to represent the respective total number of objects in each sample.

further corrections in terms of area completeness to calculate the specific frequency. The completeness-corrected number of UCDs with $M_{V}<-11 \mathrm{mag}$ is therefore $37 \pm 10$ in the central Centaurus region, with a corresponding reference specific frequency of $S_{\mathrm{N}, \mathrm{UCD}}^{*} \simeq 13.5 \pm 4.5$, and a final corrected value of $S_{\mathrm{N}, \mathrm{UCD}}=5.1 \pm 1.7$. For comparison, the GC specific frequency of NGC 4696 is $S_{\mathrm{N}, \mathrm{GC}}=7.3 \pm 1.5$ (Mieske et al. 2005), fully consistent with the value for UCDs.

\subsection{Hydra}

For the Hydra cluster we base our estimate of $S_{\mathrm{N}, \mathrm{UCD}}$ on the recent VIMOS spectroscopic study presented in Misgeld et al. (2011). The assumed distance modulus of Hydra is $33.37 \mathrm{mag}$ (Misgeld et al. 2011). We restrict our considerations to a radius of $r=5^{\prime}$ from the cluster center $(60 \mathrm{kpc})$, adopted to be at the position of the central cD galaxy NGC 3311. In Misgeld et al. (2011), we found 33 UCDs with $M_{V}<-11 \mathrm{mag}$ in this region. The projected positions of those UCDs are indicated in Fig. 2, along with those for Fornax and Centaurus. The overall completeness in terms of slit allocation is $63 \%$ for this survey. Due to the superposition of several survey pointings towards the central Hydra cluster, the slit allocation completeness in the central 5 arcmin is slightly higher, at $70 \pm 5 \%$. The area coverage within the central 5 arcmin is almost complete, at about 95\% (Figs. 1 and 7 of Misgeld et al. 2011). Together, this implies an overall survey completeness of $\sim 66 \%$, and hence gives a total number of $\sim 50 \pm 10$ UCDs. For calculating the specific frequency, we adopt an apparent magnitude of $V=10.9$ mag for NGC 3311 and $V=11.8$ for NGC 3309 (Misgeld et al. 2009), the two main galaxies in the central 5 arcmin of Hydra. Using as distance modulus 33.37 (Misgeld et al. 2011), we find $M_{V}=-22.9 \mathrm{mag}$ as total luminosity of the two galaxies. This yields a reference specific UCD frequency of $S_{\mathrm{N}, \mathrm{UCD}}^{*} \simeq 22 \pm 8$ for the Hydra cluster. Assuming a GCLF width of $\sigma=1.30 \mathrm{mag}$ (corresponding to the $M_{V}=-22.5$ mag of the dominant gE NGC 3311), we obtain a final corrected value of $S_{\mathrm{N}, \mathrm{UCD}}=12.5 \pm 4.3$. For comparison, the GC specific frequency of NGC 3311 within the inner $40 \mathrm{kpc}$ is $S_{\mathrm{N}, \mathrm{GC}}=12.5 \pm 1.5$ (Wehner et al. 2008), which agrees very well with the UCD value.

\subsection{Group environments}

\subsubsection{Local Group}

In the Milky Way there is no compact stellar system with $M_{V}<$ $-11 \mathrm{mag}$, and also for M31 it is at most two to three objects (Harris et al. 1996; Barmby et al. 2000; Huxor et al. 2011) ${ }^{3}$. We therefore use the equivalent definition of $S_{\mathrm{N}, \mathrm{UCD}}$ based on the limiting mass of $2 \times 10^{6} M_{\odot}\left(M_{V}=-10.25 \mathrm{mag}\right)$, with $S_{\mathrm{N}, \mathrm{UCD}}=$ $N_{\mathrm{UCD}} 10^{\left(0.4 M_{V, \text { host }}+20\right)} c_{\mathrm{W}}$. According to Harris et al. (1996) there is one compact stellar system in the Milky Way with $M_{V}<$ $-10.25 \mathrm{mag}$, namely $\omega$ Cen. For M 31 , the photometry of GCs is affected by both internal and foreground reddening, which makes the assessment more difficult (e.g. Galleti et al. 2004; Huxor et al. 2011). Huxor et al. (2011) find an absolute magnitude distribution for M31 GCs which peaks at $M_{V}=-7.9 \mathrm{mag}$, so is 0.5 mag brighter than the typical turnover magnitude. Their resulting GC luminosity distribution (Fig. 2 of their paper), suggests about $\sim 15 \mathrm{GCs}$ in M 31 with $M_{V}<-10.25$ mag. However, these authors indicate that uncertainties in internal extinction can heavily influence those results. Given these uncertainties, a conservative lower limit on the number of GCs in M31 may be obtained by shifting the overall magnitude distribution of M 31

${ }^{3}$ M 32 is excluded from this consideration. 
GCs 0.5 mag faintwards to match the typical turnover magnitude (and that of the Milky Way) of $M_{V} \sim-7.4$ mag. Doing so, the number of compact stellar systems with $M_{V}<-10.25 \mathrm{mag}$ reduces to $\sim 7-8$.

As a sanity check of these numbers, we furthermore consider the NIR Ks-band luminosities of the brightest M31 GCs from the revised Bologna Catalogue by Galleti et al. (2004), which are largely unaffected by reddening. Adopting a typical $(V-K)=2.5 \mathrm{mag}$ for GCs (Bruzual \& Charlot 2003 assuming $10 \mathrm{Gyr}$ and $[\mathrm{Fe} / \mathrm{H}]=-1 \mathrm{dex})$ and a distance modulus of 24.47 mag to M31 (McConnachie et al. 2005), we obtain an apparent limiting magnitude of $m_{K} \lesssim 11.7 \mathrm{mag}$ for UCDs in M31. In the revised Bologna Catalogue we find ten objects with $m_{K}<11.7 \mathrm{mag}$. This is within the range of the numbers determined from optical data above.

We thus adopt a final value of ten UCDs with $M_{V}<-10.25 \mathrm{mag}$ in M31, and one UCD in the Milky Way. We adopt as absolute visual magnitude for M31 $M_{V}=-21 \mathrm{mag}$ (Gil de Paz 2007). For the Milky Way, the absolute visual magnitude is quite uncertain as a stand-alone quantity. We adopt a somewhat fainter value of $M_{V}=-20.5$ mag compared to M 31, based on the approximate mass ratio between both galaxies when using the radial velocity distribution of their satellites as dynamical tracers (Watkins et al. 2010). The total absolute magnitude for the Local Group then is $M_{V}=-21.5$ mag. For the Local Group we thus obtain a reference specific frequency of $S_{\mathrm{N}, \mathrm{UCD}}^{*}=2.8 \pm 1.0$. Evaluating $c_{\mathrm{W}}$ for $M_{V}=-21 \mathrm{mag}$ (since M 31 contributes almost all of the UCDs), we get $\sigma=1.15 \mathrm{mag}$ and a final corrected value of $S_{\mathrm{N}, \mathrm{UCD}}=4.1 \pm 1.5$. For comparison, the GC specific frequency of M 31 is $S_{\mathrm{N}, \mathrm{GC}}=1.8 \pm 0.3$ (Barmby \& Huchra 2001; Gil de Paz et al. 2007), marginally lower than the UCD specific frequency at the $1.5 \sigma$ level.

\subsubsection{Hickson compact groups 22 and 90}

In a recent study by Da Rocha et al. (2011), a spectroscopic search for UCDs is performed in HCG 22 and 90 (distance modulus $32.6 \mathrm{mag}$ ) using FORS2 MXU spectroscopy in one $7 \times 7^{\prime}$ pointing $(65 \times 65 \mathrm{kpc})$. Also in this environment, no bright UCDs with $M_{V}<-11$ mag have been found, so we adopt the same definition of $S_{\mathrm{N}, \mathrm{UCD}}$ as for the Local Group. In Da Rocha et al. (2011) the specific frequency of UCDs with mass above $2 \times 10^{6} M_{\odot}$ is already calculated taking the survey completeness (Sects. 2.2 and 4.2. of that paper) into account; however, in this paper the authors adopt a definition based on the $B$-band luminosity of the host galaxy: $S_{\mathrm{N}, \mathrm{UCD}}^{*}=N_{\mathrm{UCD}} 10^{\left(0.4\left(M_{B, \mathrm{host}}+20\right)\right.}$. For this normalization, the specific frequency values are $6.3 \pm 2.1$ for HCG 22 and $2.0 \pm 1.0$ for HCG 90, for total $B$-band group luminosities of $M_{B}=-20.7$ and $M_{B}=-21.5$, respectively. For HCG 90 this includes a contribution of $\sim 35 \%$ intragroup light (Da Rocha et al. 2011). To convert this to the definition based on $V$-band presented in this paper, we assume a global $(B-V)=0.9 \mathrm{mag}$ for both group environments. This scales down the numerical values by a factor $10^{0.9 \times 0.4}$ and thus yields reference specific frequencies of $S_{\mathrm{N}, \mathrm{UCD}}^{*}=2.7 \pm 1.2$ and $S_{\mathrm{N}, \mathrm{UCD}}^{*}=0.9 \pm 0.5$ for HCG 22 and HCG 90. Including the correction factor $c_{\mathrm{w}}$ with $\sigma=1.15 \mathrm{mag}$ in both groups (main galaxy luminosities of $M_{V}=-21.1 \mathrm{mag}$ ), we obtain final values of $S_{\mathrm{N}, \mathrm{UCD}}=3.9 \pm 1.6$ and $S_{\mathrm{N}, \mathrm{UCD}}=1.3 \pm 0.7$ for $\mathrm{HCG} 22$ and HCG 90, respectively. The value for HCG 90 would increase to $S_{\mathrm{N}, \mathrm{UCD}} \sim 2 \pm 1$ when excluding the intragroup light component from the total group luminosity.

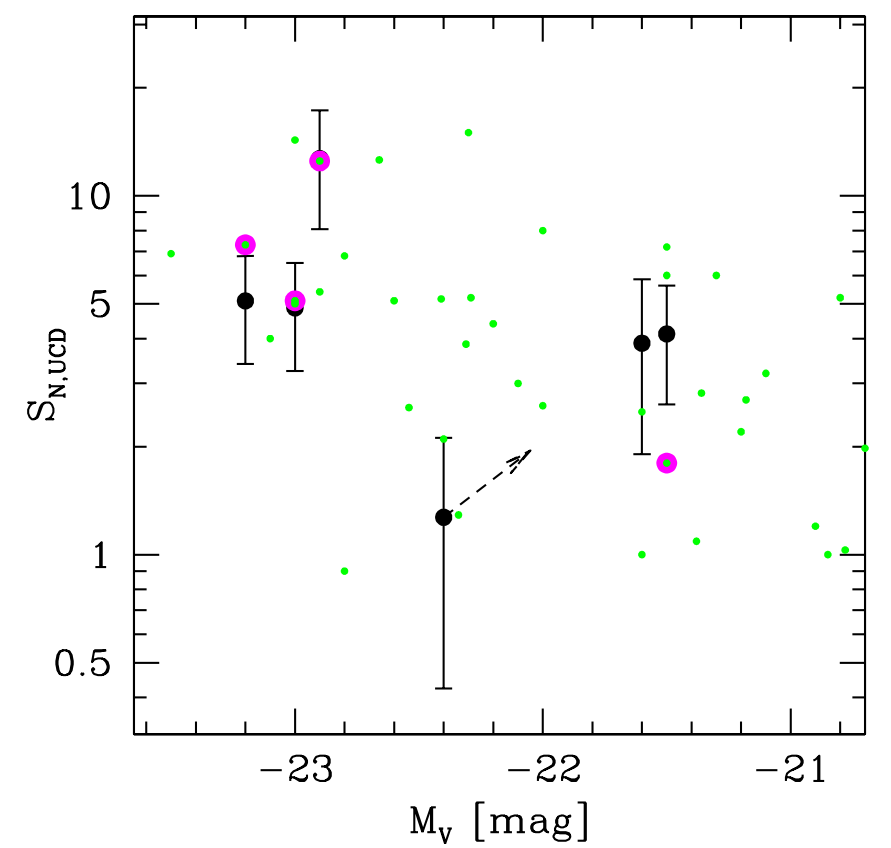

Fig. 6. Specific frequency of UCDs. The large filled circles with error bars indicate the UCD specific frequency $S_{\mathrm{N}, \mathrm{UCD}}$ as derived in the text for six different environments. These are, from bright to faint host galaxy luminosities: Centaurus, Fornax, Hydra, HCG90, HCG22, Local Group. See also Table 1. Large magenta circles are the GC specific frequencies $S_{\mathrm{N}, \mathrm{GC}}$ in the investigated environments, see text for details estimates for the HCGs are not available. Small (green) dots are literature GC specific frequencies $S_{\mathrm{N}, \mathrm{GC}}$ over a range of environments (Dirsch et al. 2003; Mieske et al. 2005; Peng et al. 2008 and references therein). The dashed arrow for HCG90 indicates the location of this data point if the $35 \%$ intragroup light component (see text) would not be considered for the total luminosity.

\subsection{Global picture of UCD specific frequencies}

The calculations performed in the previous sections regarding specific frequencies are summarized in Table 1 and in Fig. 6, which shows the UCD specific frequency $S_{\mathrm{N}, \mathrm{UCD}}$ as derived for the six different environments. For comparison, it also shows the GC specific frequency $S_{\mathrm{N}, \mathrm{GC}}$ in the respective environments. Data on $S_{\mathrm{N}, \mathrm{GC}}$ for the HCGs are not available. We also indicate literature globular cluster specific frequencies $S_{\mathrm{N}, \mathrm{GC}}$ (Dirsch et al. 2003; Mieske et al. 2005; Peng et al. 2008, and references therein) over a range of environments. The GC specific frequencies rise slightly for the brightest host galaxy magnitudes $M_{V} \lesssim-23 \mathrm{mag}$, which has been noted in a number of previous studies (e.g. Kundu \& Whitmore 2001; Brodie \& Strader 2006, and references therein; Jordán et al. 2006; Peng et al. 2008; Georgiev et al. 2010).

Overall, the specific frequencies of UCDs match those of GCs remarkably well. For the four environments where both $S_{\mathrm{N}, \mathrm{UCD}}$ and $S_{\mathrm{N}, \mathrm{GC}}$ are available, we find an average of $S_{\mathrm{N}, \mathrm{UCD}}=$ $6.7 \pm 2.0$ vs. $S_{\mathrm{N}, \mathrm{GC}}=6.7 \pm 2.2$. The mean over all six environments is $S_{\mathrm{N}, \mathrm{UCD}}=5.3 \pm 1.7$, fully consistent with the mean $S_{\mathrm{N}, \mathrm{GC}}=5.0 \pm 0.7$ averaged over all galaxies in the host magnitude range $M_{V}<-21 \mathrm{mag}$. We find mild evidence that UCDs have a higher specific frequency in more luminous hosts, as also seen for GCs (e.g. Peng et al. 2008). Adopting a dividing host galaxy luminosity at $M_{V}=-22.5 \mathrm{mag}$ (galaxy cluster vs. galaxy group), we find for UCDs that $S_{\mathrm{N}, \mathrm{UCD} \text {,bright }}=7.6 \pm 2.6$ while $S_{\mathrm{N}, \mathrm{UCD} \text {,faint }}=3.1 \pm 0.9$. This difference of about a factor of 2-3 
is formally different from zero at the $1.6 \sigma$ level. For GCs we find $S_{\mathrm{N}, \mathrm{GC} \text {,bright }}=6.4 \pm 1.4$ and $S_{\mathrm{N}, \mathrm{GC} \text {,faint }}=4.3 \pm 0.8$.

We note that UCDs have also been detected in the Virgo and Coma clusters (e.g. Jones et al. 2006; Chiboucas et al. 2010a), but the respective survey designs do not allow accurate determination of the UCD specific frequency. To solidify the suggested increase in UCD frequency in high-luminosity and denser environments, the results of homogeneous spectroscopic surveys down to $M_{V} \simeq-10$ to -11 mag in both Virgo and Coma will be very helpful.

\section{Number of UCDs compared to number of possible progenitor galaxies}

As outlined in the Introduction, there are two main formation channels for UCDs discussed in the literature, that of massive star clusters and that of tidally stripped dwarf galaxies (e.g. Zinnecker 1988; Hilker et al. 1999b; Drinkwater et al. 2000; Bekki et al. 2003; Goerdt et al. 2008). In the following we discuss the latter scenario, and use the result of the previous section to constrain its importance. The general idea is that an overabundance of UCDs with respect to GCs would indicate there is a second formation channel. An upper limit for such an overabundance of UCDs with respect to GCs can be estimated from the ratio of average UCD-to-GC specific frequencies and its error bars: $\frac{6.7 \pm 2.0}{67 \pm 22}=1.00 \pm 0.44$. The error bars of this ratio suggest that no more than $50 \%$ of UCDs may be formed via tidally stripped dwarf galaxies ( $2 \sigma$ limit).

It is worth noting that the luminosity function of dwarf galaxy nuclei is offset by two to three mag brighter with respect to their GC systems (Lotz et al. 2001; Mieske et al. 2004). Assuming a Gaussian LF, an additional $\sim 1 \%$ of objects with a LF peaking two to three mag brighter than average GCs double the number counts in the UCD luminosity regime.

In the following we compare the number of UCDs in Hydra, Centaurus, Fornax to the number of known dwarf galaxies in the same area whose luminosities are in the range expected for UCD progenitors. The null hypothesis is that all UCDs are formed via tidal threshing of nucleated dwarf galaxies (Bekki et al. 2003). Under this hypothesis we assess what fraction of a primordial dwarf galaxy population would need to have already been disrupted to account for all UCDs.

Bekki et al. (2003) perform simulations of tidal stripping of nucleated dwarf galaxies to investigate this formation channel for UCDs. They adopt a mass fraction of a few percent of the central nucleus compared to the stellar envelope of the host dwarf galaxy (a difference of 3-4 mag). The recent observational studies performed in the course of the ACS Virgo Cluster Survey (e.g. Côté et al. 2006) suggest, however, that the mass fraction of nuclei is one order of magnitude less, about $0.3 \%$ or $6-7$ mag.

To estimate the luminosity range for possible UCD progenitors, we first define as starting point the luminosity range of UCDs themselves to be $-13.5<M_{V}<-11 \mathrm{mag}$. For the UCD progenitors, we then adopt a magnitude difference of $6 \pm 1$ mag, which yields a magnitude range $-20.5<M_{V}<-16$ for potential UCD progenitor galaxies.

The left panel of Fig. 2 shows the map of the projected positions of UCDs in the central $50 \mathrm{kpc}$ of the Hydra, Fornax and Centaurus clusters, adopting as UCD limit $M_{V}<-11 \mathrm{mag}$ (see previous section). In the right panel of Fig. 2 we show in addition the projected positions of the potential UCD progenitor galaxies, that is, those galaxies with $-20.5<M_{V}<$ $-16 \mathrm{mag}$. For Hydra, the galaxy positions and magnitudes are taken from a merging of the FORS2 Hydra Cluster Catalog (Misgeld et al. 2009) and the spectroscopic study of Christlein $\&$ Zabludoff (2003). For Centaurus, the galaxies are from a merging of the Centaurus Cluster Catalog (Stein et al. 1997) and the FORS2 photometric study of Misgeld et al. (2008). For the Fornax cluster, the galaxies are from a merging of the Fornax Cluster Catalogue (Ferguson \& Sandage 1988) and the photometric study of Mieske et al. (2007) performed with IMACS@Magellan.

For the right panel of Fig. 2 we assume that the galaxy catalogs for cluster members in the three environments are complete within the magnitude range considered. The UCD searches in the same area have a completeness significantly below 100\% (see previous section). Averaging over the three clusters and weighting with the respective number of detected UCDs, we obtain an estimate of $\sim 62 \pm 5 \%$ for the completeness of the UCD search ${ }^{4}$. In the right panel of Fig. 2 we therefore plot only $62 \%$ of all eligible progenitor galaxies in this figure, which allows a direct comparison of the number counts. We furthermore highlight the very brightest subsample of UCDs, adopting a (somewhat arbitrary) limit of $M_{V}<-12 \mathrm{mag}$.

This plot shows that, within the same projected radius, the number of UCDs with $M_{V}<-11 \mathrm{mag}$ is a factor of 6-7 larger than the number of existing possible progenitor galaxies. Under the null hypothesis that all UCDs are created by tidal stripping, this plot suggests that at least $\frac{54}{54+9} \simeq 85 \%$ of primordial dwarf galaxies in the central $\sim 50-70 \mathrm{kpc}$ of the considered galaxy clusters would have had to be tidally disrupted already. The situation is naturally less extreme when restricting to the very brightest UCDs with $M_{V}<-12$ mag. For such a scenario one would expect a more modest two thirds of primordial dwarfs to have been tidally disrupted by now.

In the right panel Fig. 5, we present a more extended look at the situation in the Fornax cluster, given the large available UCD database. We show a comparison of the cumulative radial distribution of UCDs and progenitor dwarf galaxies out to $200 \mathrm{kpc}$ clustercentric distance. The curves are based on Fig. 3, and have been renormalized to represent the relative size of each subsample. It is again very clear from this plot that UCDs vastly outnumber present-day progenitor galaxies. Compared to the full UCD sample down to $M_{V}<-10.25 \mathrm{mag}$, the number of progenitor dwarfs is only a few percent. UCDs are much more centrally clustered. If indeed a significant amount of UCDs were formed from tidal processes, then the tidal disruption of low-mass dark matter halos in the central Fornax cluster must have been extremely efficient ( $\gtrsim 90 \%)$.

We note that the particular choice of 6 mag for the magnitude difference between progenitor and UCD influences the considerations above to some extent, since the galaxy LF increases towards fainter luminosities. A smaller magnitude difference between UCDs and progenitors will slightly increase the number of eligible progenitor galaxies. When assuming a mean magnitude difference of 4 mag instead of 6 mag between UCDs and progenitors, the number of progenitors changes from 9 to 12 within $50 \mathrm{kpc}$ for the joint Hydra-Fornax-Centaurus sample. Still, this is far from the number of existing UCDs.

Bekki et al. (2003) performed a theoretical study tailored precisely to the case of UCD formation via tidal stripping of dwarf galaxies. They determine a "threshing radius", postulating that dwarf galaxies with orbital pericenters within that radius would become tidally transformed to UCDs. This concept is also employed by the analytical study in Thomas et al. (2008). For

${ }^{4}(0.712+0.518 .5+0.6633) / 63.5=0.62$. 
typical dwarf galaxy luminosities/masses, Bekki et al. predict the threshing radius to be in the range $50-100 \mathrm{kpc}$ for the Fornax cluster, and $70-150 \mathrm{kpc}$ for the Virgo cluster (Fig. 7 of their paper). Therefore, the strong central clustering of UCDs (Fig. 3) and the implied scenario where $\gtrsim 90 \%$ of primordial dwarf galaxies in the central $\sim 50-70 \mathrm{kpc}$ are disrupted, are in qualitative agreement with this model.

There are some more recent studies in the literature considering the tidal disruption of satellite galaxies (e.g. Henriques et al. 2008; Henriques \& Thomas 2010; Yang et al. 2009) and their contribution to the intracluster light. However, those studies were not specifically tailored to address the tidal stripping scenario for UCD formation.

Yang et al. (2009) argue that tidal disruption of satellites will be more efficient for high ratios between satellite and host halo mass. At the same time, they show that the stellar mass contributed to the intracluster medium by dissolving satellites is higher for massive host halos $\sim 10^{14} M_{\odot}$ than for lower mass halos $\sim 10^{12} M_{\odot}$.

Henriques et al. (2008) estimate that about one half of satellite galaxies get disrupted and/or accreted to their host halos and that $10 \%$ of the overall cluster light is found in the intracluster medium, originating from tidally stripped stars. In their most recent paper, Henriques \& Thomas (2010) improve their treatment of tidal disruption. In turn they revise the intra-cluster light fraction provided by disrupted galaxies upwards to $30 \%$. Their models predict $40 \%$ of the satellites with masses between $10^{9}$ and $10^{10} M_{\odot}$ to be tidally disrupted. This fraction increases to $57 \%$ when considering satellites that have already lost their dark matter halo ("orphan satellites") in previous interactions (Henriques 2011, priv. comm.).

This $\sim 50 \%$ global disruption fraction is lower than the fraction of $\sim 90 \%$ needed for the limiting case that half of today's UCDs originate from tidal processes (see above). Radial trends of disruption efficiency would still be expected (Bekki et al. 2003), such that this disruption fraction can be considered a lower limit to the value applicable to the clustercentric areas studied in this paper.

\section{Summary and conclusions}

In this paper we have proposed a definition for the specific frequency of UCDs, which we denote as $S_{\mathrm{N}, \mathrm{UCD}}$. We adopt the following functional form:

$$
S_{\mathrm{N}, \mathrm{UCD}}=N_{\mathrm{UCD}} 10^{0.4\left(M_{V, \mathrm{host}}-M_{V, 0}\right)} c_{\mathrm{W}} .
$$

This definition normalizes the number of UCDs in a given environment to a unit host galaxy luminosity $M_{V \text {,host }}$, analogous to the definition of the specific frequency $S_{\mathrm{N}, \mathrm{GC}}$ for GCs. The premise of our definition is that if UCDs follow the extrapolation of the GCLF to bright magnitudes, then $S_{\mathrm{N}, \mathrm{UCD}}=S_{\mathrm{N}, \mathrm{GC}}$. This premise defines the value of the zero point $M_{V, 0}$. Considering UCDs as compact stellar systems with $M_{V}<-10.25 \mathrm{mag}$, we find that the value of $M_{V, 0}=-20$ mag fulfills the above premise. For the case of extragalactic surveys with a brighter completeness limit $M_{V}<-11.0 \mathrm{mag}$, we need to adopt $M_{V, 0}=-22 \mathrm{mag}$. The term $c_{\mathrm{W}}$ is introduced to correct the specific frequency for the well known systematic variation of the GCLF width $\sigma$ with host galaxy magnitude. Details of this correction are outlined in Sect. 2. In Sect. 3 we apply our proposed definition of $S_{\mathrm{N}, \mathrm{UCD}}$ to results of spectroscopic UCD searches performed by our group in the Fornax, Hydra and Centaurus galaxy clusters, and two Hickson Compact Groups. We also include the Local Group.
Our main finding is that the specific frequencies derived for UCDs match those of GCs very well. For four of the six investigated environments, there are GC specific frequency measurements available, allowing a direct comparison. For those four environments we find a mean $S_{\mathrm{N}, \mathrm{UCD}}=6.7 \pm 2.0$, vs. $S_{\mathrm{N}, \mathrm{GC}}=6.7 \pm 2.2$. The ratio of UCD-to-GC specific frequency is therefore $\frac{6.7 \pm 2.0}{6.7 \pm 2.2}=1.00 \pm 0.44$. The mean $S_{\mathrm{N}, \mathrm{UCD}}$ of all six investigated environments is $5.3 \pm 1.7$, in agreement with the average GC specific frequency of $5.0 \pm 0.7$ of the available literature data for the corresponding host magnitude range $M_{V}<-21 \mathrm{mag}$. Our findings are consistent with the hypothesis that most UCDs are formed by the same process as the overall GC population, and with a similar formation efficiency to GCs.

We also present an extension of our analysis in the Fornax cluster by using the large available data set of spectroscopically confirmed UCDs and GCs. This literature data set comprises about 180 confirmed UCDs with $M_{V}<-10.25 \mathrm{mag}$. We find that the specific frequencies of UCDs around giant galaxies and in the intracluster space are consistent with their being drawn from the bright tail of the GCLF. There is still room for a possible UCD overabundance in the intracluster space by a factor of $\sim 2$, given the incompleteness in spectropscopic coverage in those regions. We do not find significant evidence of a different spatial distribution between UCDs and GCs.

It has been proposed that the present-day population of UCDs is indeed a superposition of sources formed via tidal stripping of dwarf galaxies and sources formed in the same process as the main GC population (e.g. Haşegan et al. 2005; Mieske et al. 2006; Chilingarian et al. 2011; Norris et al. 2011; da Rocha et al. 2011). The error bars of the specific frequencies derived for UCDs suggest $\sim 50 \%$ as an upper limit for the importance of the dwarf galaxy channel. We show in Sect. 4 that this would require at least $\gtrsim 90 \%$ of primordial dwarf galaxies in the central $\sim 50-70 \mathrm{kpc}$ of the considered galaxy clusters to have already been disrupted. If indeed a significant amount of UCDs were formed from tidal processes, then the tidal stripping of stars from low-mass dark matter halos in the central Fornax cluster has been extremely efficient.

We conclude that the number counts of UCDs are fully consistent with them being the bright tail of the GC population. From a statistical point of view there is no need to invoke an additional formation channel. The statistical error bars constrain the fraction of tidally stripped dwarfs to not more than $50 \%$ of UCDs.

Acknowledgements. We thank the anonymous referee for very useful comments and suggestions that helped to improve the paper. We thank Bruno Henriques for providing us with details on the number fraction of disrupted satellite galaxies in their models. I.M. acknowledges support through DFG grant BE1091/13-1.

\section{References}

Barmby, P., Huchra, J. P., Brodie, J. P., et al. 2000, AJ, 119, 727

Barmby, P., Huchra, J. P., \& Brodie, J. P. 2001, AJ, 121, 1482

Bassino, L. P., Faifer, F. R., Forte, J. C., et al. 2006a, A\&A, 451, 789

Bassino, L. P., Richtler, T., \& Dirsch, B. 2006b, MNRAS, 367, 156

Bekki, K., \& Freeman, K. C. 2003, MNRAS, 346, L11

Bekki, K., Couch, W. J., Drinkwater, M. J., \& Shioya, Y. 2003, MNRAS, 344, 399

Bergond, G., Athanassoula, E., Leon, S., et al. 2007, A\&A, 464, L21

Brodie, J., \& Strader, J. 2006, ARA\&A, 44, 193

Bruzual, G., \& Charlot, S. 2003, MNRAS, 344, 1000

Carraro, G., \& Lia, C. 2000, A\&A, 357, 977

Chandar, R., Whitmore, B., \& Lee, M. G. 2004, ApJ, 611, 220

Chiboucas, K., Brent Tully, R., Marzke, R. O., et al. 2010a, EAS, 48, 225

Chiboucas, K., Tully, R. B., Marzke, R. O., et al. 2010b, ApJ, 723, 251

Chilingarian, I. V., Mieske, S., Hilker, M., \& Infante, L. 2011, MNRAS, 412, 1627 
Christlein, D., \& Zabludoff, A. 2003, ApJ, 591, 764

Côté, P., Piatek, S., Ferrarese, L., et al. 2006, ApJS, 165, 57

Da Costa, G. S., \& Coleman, M. G. 2008, AJ, 136, 506

Dabringhausen, J., Hilker, M., \& Kroupa, P. 2008, MNRAS, 386, 864

Da Rocha, C., Mieske, S., Georgiev, I. Y., et al. 2011, A\&A, 525, A86

Dirsch, B., Richtler, T., Geisler, D., et al. 2003, AJ, 125, 1908

Drinkwater, M. J., Jones, J. B., Gregg, M. D., \& Phillipps, S. 2000, PASA, 17, 227

Drinkwater, M. J., Gregg, M. D., Hilker, M., et al. 2003, Nature, 423, 519

Evstigneeva, E. A., Drinkwater, M. J., Jurek, R., et al. 2007, MNRAS, 378, 1036

Fellhauer, M., \& Kroupa, P. 2002, MNRAS, 330, 642

Fellhauer, M., \& Kroupa, P. 2005, MNRAS, 359, 223

Ferguson, H. C., \& Sandage, A. 1988, AJ, 96, 152

Ferrarese, L., Ford, H. C., Huchra, J., et al. 2000, ApJS, 128, 431

Firth, P., Drinkwater, M. J., Evstigneeva, E. A., et al. 2007, MNRAS, 382, 1342

Firth, P., Drinkwater, M. J., \& Karick, A. M. 2008, MNRAS, 389, 1539

Frank, M., Hilker, M., Mieske, S., et al. 2011, MNRAS, 414, L70

Galleti, S., Federici, L., Bellazzini, M., Fusi Pecci, F., \& Macrina, S. 2004, A\&A, 416, 917

Georgiev, I. Y., Puzia, T. H., Goudfrooij, P., \& Hilker, M. 2010, MNRAS, 406, 1967

Gieles, M., Baumgardt, H., Heggie, D. C., \& Lamers, H. J. G. L. M. 2010, MNRAS, 408, 16

Gil de Paz, A., Boissier, S., Madore, B. F., et al. 2007, ApJS, 173, 185

Goerdt, T., Moore, B., Kzantzidis, S., et al. 2008, MNRAS, 385, 2136

Goudfrooij, P., Strader, J., Brenneman, L., et al. 2003, MNRAS, 343, 665

Gregg, M., Drinkwater, M. J., Evstigneeva, E., et al. 2009, AJ, 137, 498

Harris, W. E. 1991, ARA\&A, 29, 543

Harris, W. E. 1996, AJ, 112, 1487

Harris, W. E., \& van den Bergh, S. 1981, AJ, 86, 1627

Haşegan, M., Jordán, A., Côté, P., et al. 2005, ApJ, 627, 203

Henriques, B. M., \& Thomas, P. A. 2010, MNRAS, 403, 768

Henriques, B. M., Bertone, S., \& Thomas, P. A. 2008, MNRAS, 383, 1649

Hilker, M. 2011, EAS PS, 48, 219

Hilker, M., \& Richtler, T. 2000, A\&A, 362, 895

Hilker, M., Infante, L., Vieira, G., Kissler-Patig, M., \& Richtler, T. 1999a, A\&AS, 134, 75

Hilker, M., Infante, L., \& Richtler, T. 1999b, A\&AS, 138, 55

Huxor, A. P., Ferguson, A. M. N., Tanvir, N. R., et al. 2011, MNRAS, 414, 770

Jordán, A., McLaughlin, D. E., Côté, P., et al. 2006, ApJ, 651, L25

Jordán, A., McLaughlin, D. E., Côté, P., et al. 2007, ApJS, 171, 101

Jones, J. B., Drinkwater, M. J., Jurek, R., et al. 2006, AJ, 131, 312

Kissler-Patig, M., Kohle, S., Hilker, M., et al. 1997, A\&A, 319, 470
Kissler-Patig, M., Grillmair, C. J., Meylan, G., et al. 1999, AJ, 117, 1206

Kundu, A., \& Whitmore, B. 2001, AJ, 121, 2950

Lotz, J. M., Telford, R., Ferguson, H. C., et al. 2001, ApJ, 552, 572

Majewski, S. R., Patterson, R. J., Dinescu, D. I., et al. 2000, The Galactic Halo: From Globular Cluster to Field Stars, Proceedings of the 35th Liege International Astrophysics Colloquium, held 5-8 July, 1999, ed. A. Noels, P. Magain, D. Caro, E. Jehin, G. Parmentier, \& A. A. Thoul (Liege, Belgium: Institut d'Astrophysique et de Geophysique), 619

McConnachie, A. W., Irwin, M. J., Ferguson, A. M. N., et al. 2005, MNRAS, 356, 979

Mieske, S., Hilker, M., \& Infante, L. 2002, A\&A, 383, 832

Mieske, S., Hilker, M., \& Infante, L. 2004, A\&A, 418, 445

Mieske, S., Hilker, M., \& Infante, L. 2005, A\&A, 438, 103

Mieske, S., Hilker, M., Infante, L., \& Jordán, A. 2006, AJ, 131, 2442

Mieske, S., Hilker, M., Jordán, A., Infante, L., \& Kissler-Patig, M. 2007, A\&A 472, 111

Mieske, S., Hilker, M., Jordán, A., et al. 2008, A\&A, 487, 921

Mieske, S., Hilker, M., Misgeld, I., et al. 2009, A\&A, 498, 705

Minniti, D., Kissler-Patig, M., Goudfrooij, P., \& Meylan, G. 1998, AJ, 115, 121

Misgeld, I., Mieske, S., \& Hilker, M. 2008, A\&A, 486, 697

Misgeld, I., Hilker, M., \& Mieske, S. 2009, A\&A, 496, 683

Misgeld, I., Mieske, S., Hilker, M., et al. 2011, A\&A, 531, A4

Murray, N. 2009, ApJ, 691, 946

Norris, M. A., \& Kannappan, S. J. 2011, MNRAS, 414, 739

Noyola, E., Gebhardt, K., \& Bergmann, M. 2008, ApJ, 676, 1008

Peng, E. W., Jordán, A., Côté, P., et al. 2008, ApJ, 681, 197

Phillipps, S., Drinkwater, M. J., Gregg, M. D., \& Jones, J. B. 2001, ApJ, 560 201

Rhode, K., \& Zepf, S. 2004, AJ, 127, 302

Schuberth, Y., Richtler, T., Hilker, M., et al. 2010, A\&A, 513, A52

Stein, P., Jerjen, H., \& Federspiel, M. 1997, A\&A 1997, 327, 952

Thomas, P. A., Drinkwater, M. J., \& Evstigneeva, E. 2008, MNRAS, 389, 102

Watkins, L. L., Evans, N. W., \& An, J. H. 2010, MNRAS, 406, 264

Wehner, E., Harris, W., Whitmore, B., Rothberg, B., \& Woodley, K. 2008, ApJ, 681,1233

Yang, X., Mo, H. J., \& van den Bosch, F. C. 2010, ApJ, 693, 830

Zinnecker, H., Keable, C. J., Dunlop, J. S., Cannon, R. D., \& Griffiths, W. K. 1988, in The Harlow Shapley Symposium on Globular Cluster Systems in Galaxies, Proceedings of the 126th Symposium of the International Astronomical Union, held in Cambridge, Massachusetts, USA August 25-29, 1986, ed. J. E. Grindlay, \& A. G. Davis Philip (Dordrecht: Kluwer Academic Publishers), 603 